\title{
Integrated Care in Europe: Time to Get it Together?
}

\author{
Livio Garattini ${ }^{1}$ [ $\cdot$ Marco Badinella Martini ${ }^{1} \cdot$ Alessandro Nobili $^{1}$
}

Accepted: 4 August 2021 / Published online: 30 August 2021

(C) The Author(s), under exclusive licence to Springer Nature Switzerland AG 2021

\section{Introduction}

Nowadays integrated care (IC) has become a term adopted across the world underpinning a positive attitude toward defragmentation of service provision inside health and social systems [1].

Since lack of healthcare coordination is often a major problem for chronic and frail patients, integration has certainly commendable aims [2]. Striving for combining parts to form a whole, IC aims at optimizing care and treatments to patients and their caregivers [3]. However, while the principles supporting IC are simple, their implementation is more controversial [4].

As it often happens in the health literature, IC has rapidly become an 'umbrella concept' open to various interpretations [5]. After the launch of an international journal in 2000 including IC in its name, a search conducted a decade later had already found more than 175 definitions [6]. Recently, even the European office of the World Health Organization (WHO) issued a working document to provide conceptual clarity on IC models [7].

Here, we summarize the current narrative and the main issues of IC. Then, we draw lessons for Europe and finally put forward a few recommendations in the perspective of a long-term harmonization in the European Union (EU).

\section{Definition and Jargon}

The growing number of IC definitions is somehow related to the increasing domains of applications. In addition to general IC definitions [8], many IC models have been recently focused on specific groups of patients, often older adults and frail subjects. This reflects the major current challenge

Livio Garattini

livio.garattini@marionegri.it

1 Institute for Pharmacological Research Mario Negri IRCCS, Ranica, BG, Italy of health systems in highly developed countries, i.e. the increasing demand and proportion of healthcare expenditures induced by ageing chronic patients with both physical and cognitive problems [9]. So, the most recent conceptual essence of IC is the creation of social and health care delivery systems aimed at spanning the borders and improving the communication among services in primary, secondary and tertiary care [10].

Beyond trendy terms like patient-centered care, often cited as the key aim for IC compared to the traditional disease-centered care provided by single health services $[8,11]$, some terms are more specific to the IC concept. In addition to the original IC triple aim of improving people health, enhancing patient experience and reducing health care costs [8, 9], the quadruple aim of improving the work life of health care professionals has been added later on [12].

As in any other supply chain, integration can be either horizontal or vertical according to microeconomics [4]. The former occurs when IC is applied to various services delivered at the same stage (e.g. hospital wards in tertiary care), the latter when IC brings together services delivered at different stages (e.g. general practices in primary care and hospital wards in secondary care) [13]. Also, IC strategies can target three levels of service provision according to social sciences: micro-level for individual patients, mesolevel for groups of patients (e.g. older multi-morbid adults) and macro-level for the whole populations [4, 8].

Because IC interventions usually involve multi-disciplinary teams, case management is considered the new role to ensure coordination and communication among different professionals, regularly keep in touch with patients, and finally reduce healthcare and social costs [1, 9, 14].

To conclude, although no single model or definition of IC fits all contexts [12], expectations on IC are still growing and IC initiatives are expected to increase throughout Europe [11]. 


\section{An Endless Debate}

The recent debate on IC is largely influenced by the mismatch between the increasing burden of health and social needs for chronic conditions and patient complexity from the demand side, and the design of healthcare systems still focused on acute care and single diseases from the supply side $[9,10,15]$. This gap might even increase health risks for multi-morbid patients when receiving conflicting recommendations and treatments from different healthcare providers [11].

During this endless period of budgetary pressures the wide debate on IC in the EU has also been affected by the different types of health systems [16]. The 'Bismarckian' systems (statutory health insurances) have by default many more players compared to the 'Beveridgian' systems (national health services). So, the former are inevitably less favorable to IC [17]. In general, when financial incentives of specific players run against IC interventions, it is hard to involve them, since these players follow their own interests. At micro level, funding is an issue to face in all IC models when trying to involve social services provided outside health systems.

IC initiatives might also reveal 'unmet needs' of multimorbid patients within and beyond health care [4, 12], eventually leading to extra costs rather than savings [3, 11]. At macro level, the ever ageing populations are likely to have put more under strain the social services of southern countries traditionally characterized by a strong family culture like Italy and Spain, where relatives are used to playing the role of informal caregivers when necessary [15].

In general, it is methodologically challenging to prove the effectiveness of IC interventions based on scientific evidence [4]. The outcome measures of organizational studies and their empirical results are hardly comparable with those of 'usual care', which in turn can vary widely at local level even within the same health system. Ultimately, this makes the design of studies on IC initiatives open to discretion and their results hardly extendable to other settings, so that any attempt to estimate 'trade-offs' between IC additional costs and potential savings seems arbitrary [18].

Healthcare services are quite peculiar from an organizational point of view, since their power structure is somehow reversed compared to the vast majority of other fields [19]. Healthcare professionals at bottom levels have greater influence over daily decision-making than those hierarchically placed at top $[1,20]$. Therefore, changes in clinical practice are more likely to be achieved thanks to managerial strategies aimed at building professionals' trust through bottom-up incremental steps, rather than through top-down hierarchical directives [21]. This hardly fits common politicians' attitude, who expect to announce fast results of eye-catching IC initiatives conducted by purposely appointed top managers [22].

Consistently, also the new emerging role of case managers for IC is open to debate [23]. Rather than adding new jobs for spanning boundaries among services, involving the existing staff might be a more effective strategy to push IC [24]. Creating a culture that aligns the existing professionals would also curtail blurring of roles and fears of job losses.

To conclude, IC is another example of a wise concept initially rising enthusiasm, then finding an insurmountable hurdle in scant clinical evidence [25], and finally leading to an endless debate [3].

\section{Lessons for Europe}

The major reasons of persisting IC weakness in Western European countries stem from arguable choices of health policy taken in the recent past. The political creed in 'market competition' is probably the most emblematic [16]. Health is a classical example of 'market failure' in economics, from both demand and supply side. So, the myth of competition is fully unjustified and required strong ideological support since the first British attempt [16]. All initiatives encouraging healthcare providers to compete are likely to discourage IC $[1,26]$. Furthermore, activity-based funding through arbitrarily fixed tariffs for services can only lead to distortions in allocating financial resources and ultimately undermine IC, discouraging coordination and synergies among providers [27].

The professional status of general practitioners is another historically rooted reason of IC weakness throughout Europe. Differently from their colleagues in hospitals, who are mainly employees, GPs are still self-employed professionals in the English and Italian NHSs too [16, 26]. Since co-location of health professionals favors IC thanks to team-working $[1,23]$, single large-scale organizations have become a pressing priority for a modern primary care [28]. Therefore, the time has come to radically change the anachronistic status of GPs, in order to strengthen IC in the future [29].

\section{Final Proposals}

Finally, we offer some recommendations to improve IC in Europe. A national health service should be indicated as the type to be preferred, since public healthcare systems facilitate IC for both funding and provision. Rather than pricing and competing, budgeting and planning should be the right culture for managing them [16]. Ideally, the 
future healthcare systems should become NHSSs by adding the second 's' of social, merging health and social budgets to bring all types of services closer together [15, 28]. To improve the supervision of NHSSs activity in a IC perspective, all health and social professionals should become employees, GPs included. This should allow the NHSSs to better cope with the big challenge of constraining political influence and administrative bureaucracy, the most serious motivation-killing threats of public sectors [15]. Striving to enhance patients' health as the primary interest of healthcare services, the NHSS organization should be inspired by systemic coordination among health and social professionals [27], adding communication and management skills to their traditional education and training [24].

In conclusion, we are convinced that the quadruple aim of IC would not be a mirage in a collaborative rather than competitive context. This should contribute to stop the current European debate around IC.

Acknowledgements LG would like to thank his friend Silvia Zebramini for her useful comments on the first draft of the manuscript.

\section{Declarations}

Funding No sources of funding were used to conduct this study or prepare this manuscript.

Conflict of interest Livio Garattini, Marco Badinella Martini and Alessandro Nobili have no conflicts of interest directly relevant to this article.

\section{References}

1. Shaw S, Rosen R, Rumbold B. What is integrated care? An overview of integrated care in NHS. London, Nuffield Trust 2011. http://www. nuffieldtrust.org.uk/sites/files/nuffield/publication/what_is_integ rated_care_research_report.

2. Damery S, Flanagan S, Combes G. Does integrated care reduce hospital activity for patients with chronic diseases? An umbrella review of systematic reviews. BMJ Open. 2016;6:e011952.

3. Goodwin N. Understanding integrated care: a complex process, a fundamental principle. Int J Integr Care. 2013;13:e011.

4. Goddard M, Mason AR. Integrated care: a pill for all ills? Int J Health Policy Manag. 2017;6(1):1-3.

5. Goodwin N. Understanding integrated care. Int J Integr Care. 2016;16(4):6.

6. Armitage GD, Suter E, Oelke ND, Adair C. Health systems integration: state of the evidence. Int J Integr Care. 2009;9(17):1-11.

7. World Health Organization. Integrated care models: an overview. Copenhagen: WHO Regional Office for Europe; 2016.

8. Briggs AM, Valentijn PP, Thiyagarajan JA, Araujo de Carvalho I. Elements of integrated care approaches for older people: a review of reviews. BMJ Open. 2018;8(4):e021194.

9. Stokes J, Riste L, Cheraghi-Sohi S. Targeting the "right" patients for integrated care: stakeholder perspectives from a qualitative study. J Health Serv Res Policy. 2018;23(4):243-51.

10. Erskine J, Castelli M, Hunter D, Hungin A. The persistent problem of integrated care in English NHS hospitals (Mayo Model). J Health Organ Manag. 2018;32(4):532-44.
11. van der Heide I, Snoeijs S, Quattrini S, Struckmann V, Hujala A, Schellevis F, Rijken M. Patient-centeredness of integrated care programs for people with multimorbidity. Results from the European ICARE4EU project. Health Policy. 2018;122(1):36-43.

12. Baxter S, Johnson M, Chambers D, et al. The effects of integrated care: a systematic review of UK and international evidence. BMC Health Serv Res. 2018;18(1):350.

13. Baltaxe E, Cano I, Herranz C, Barberan-Garcia A, Hernandez C, Alonso A, Arguis MJ, Bescos C, Burgos F, Cleries M, Contel JC, de Batlle J, Islam K, Kaye R, Lahr M, Martinez-Palli G, Miralles F, Moharra M, Monterde D, Piera J, Ríos J, Rodriguez N, Ron R, Rutten-van Mölken M, Salas T, Santaeugenia S, Schonenberg H, Solans $\mathrm{O}$, Torres G, Vargiu E, Vela E, Roca J. Evaluation of integrated care services in Catalonia: population-based and service-based real-life deployment protocols. BMC Health Serv Res. 2019;19(1):370.

14. Everink IHJ, van Haastregt JCM, Tan FES, Schols JMGA, Kempen GIJM. The effectiveness of an integrated care pathway in geriatric rehabilitation among older patients with complex health problems and their informal caregivers: a prospective cohort study. BMC Geriatr. 2018;18(1):285.

15. Mur-Veeman I, Hardy B, Steenbergen M, Wistow G. Development of integrated care in England and the Netherlands: managing across public-private boundaries. Health Policy. 2003;65(3):227-41.

16. Garattini L, Padula A. Competition in health markets: is something rotten? J R Soc Med. 2019;112(1):6-10.

17. Milstein R, Blankart CR. The Health Care Strengthening Act: the next level of integrated care in Germany. Health Policy. 2016;120(5):445-51.

18. Garattini L, Padula A, Freemantle N. Do European pharmacists really have to trespass on medicine? Eur J Health Econ. 2021;22(1):1-4.

19. Ham C. Improving the performance of health services: the role of clinical leadership. Lancet. 2003;361(9373):1978-80.

20. McDermott I, Checkland K, Moran V, Warwick-Giles L. Achieving integrated care through commissioning of primary care services in the English NHS: a qualitative analysis. BMJ Open. 2019;9(4):e027622.

21. Kodner DL, Spreeuwenberg C. Integrated care: Meaning, logic, applications, and implications - a discussion paper. Int J Integr Care 2002;2:e12.

22. Alderwick H, Dunn P, Gardner T, Mays N, Dixon J. Will a new NHS structure in England help recovery from the pandemic? BMJ. 2021;372:n248.

23. Iacobucci G. Use existing staff to drive integrated care, says report. BMJ. 2016;353:i3661.

24. Gilburt H. Supporting integration through new roles and working across boundaries. London: King's Fund. 2016. http://www.kings fund.org.uk/publications/supporting-integration-new-roles-bound aries.

25. Eyre L, Farrelly M, Marshall M. What can a participatory approach to evaluation contribute to the field of integrated care? BMJ Qual Saf. 2017;26(7):588-94.

26. Hardy B, Mur-Veeman I, Steenbergen M, Wistow G. Interagency services in England and The Netherlands. A comparative study of integrated care development and delivery. Health Policy. 1999;48(2):87-105.

27. Garattini L, Padula A. Defensive medicine in Europe: a "full circle"? Eur J Health Econ. 2020;21(2):165-70.

28. Garattini L, Badinella Martini M, Zanetti M. More room for telemedicine after COVID-19: lessons for primary care? Eur J Health Econ. 2021;22(2):183-6.

29. Das P, Naylor C, Majeed A. Bringing together physical and mental health within primary care: a new frontier for integrated care. J R Soc Med. 2016;109(10):364-6. 